\title{
NOCIONES RELACIONADAS CON LAS CUEVAS EN LA LENGUA Y COSMOVISIÓN TSELTALES
}

\author{
Notions about the Caves in the Tseltal Language and Worldview \\ Marceal Méndez-Pérez
}

Resumen: En el presente texto se estudian algunas nociones relacionadas con las cuevas entre los tseltales de Chiapas, las cuales sólo en lengua tseltal parecen tener sentido. Dichas nociones permiten conocer y comprender otras funciones importantes de las cuevas, como su carácter de entradas hacia el interior de la tierra y la montaña, o hacia el lugar llamado Inframundo.

Palabras clave: cuevas, tierra, montaña, Ajaw, naturaleza, inframundo.

Abstract: In this text we study some notions about the caves among the tseltales of Chiapas, wich only in tseltal language seems to make sense. These notions allow us to know and understand other important functions of the cave as entrance to within the earth and the mountains, to the place called Underworld.

Keywords: caves, earth, mountain, Ajaw, landscape, underworld.

Marceal Méndez Pérez, maestro en ciencias sociales y humanísticas. Investigador en el Centro Estatal de Lenguas, Arte y Literatura Indígenas (CELALI), Chiapas, México. Temas de especialización: religión indígena actual, literatura, tradición oral. Correo electrónico: petalcingo@hotmail.com.

Enviado a dictamen: 19 de abril de 2013. Aprobación: 22 de junio de 2013.

Revisiones: 1. 


\section{Introducción}

E n el presente texto se aborda uno de los componentes más importantes que dan sentido de sacralidad a la tierra y las montañas entre los actuales tseltales de Chiapas: las cuevas. Sobre éstas se dispone de abundantes estudios, por ejemplo, los escritos por Thompson (1959), Bonor (2003), Sheseña (2006), Bassie (2006) o Brady (2009). Los autores destacan el uso y las funciones que las cuevas han tenido a lo largo de la historia mesoamericana. A este esfuerzo por comprender la naturaleza sagrada de las cuevas, queremos agregar un estudio de algunos de sus atributos que, debido a su carácter profundamente simbólico, salen a colación sólo en la intimidad de las conversaciones con los ancianos tseltales. Para ello, los conceptos de "racionalidad", planteado por Winch (1979), y de "revalorización de las categorías culturales", propuesto por Sahlins (1997), han sido de mucha utilidad para identificar, describir y comprender tanto las características, como los cambios de los atributos y de nociones relacionados con las cuevas. En términos específicos de la lengua tseltal, nos referimos a las nociones de kuxul, yajnib chanbajlam, skuxibyo'tan Ajaw, sti'nail o sbentanail wits, lok'ib ik', yich'u ik' bajlumilal, tajnib ch'ulelaletik y smajkibjnutsujel ts'i'etik, las cuales explicaremos más adelante.

Cabe destacar que el presente estudio no estuvo en su preparación exento de complicaciones en el trabajo de campo, las cuales, aunque no impidieron su realización, sí limitaron las posibilidades de entender y detallar las caracterizaciones y, por tanto, la dinámica de las cuevas como espacios sagrados en la práctica religiosa contemporánea. En general, fueron tres las dificultades:

1. Debido al abandono de las prácticas rituales por parte de algunas personas y oficiantes, la información sobre las cuevas es narrada por buena parte de los entrevistados con cierto desánimo y algo de nostalgia; en consecuencia, los datos que se obtienen de las entrevistas son generales. Es decir, las caracterizaciones obtenidas pueden corresponder a todos los espacios sacros y coinciden con los de cualquier otro pueblo, por muy distante que se encuentre. Se han olvidado, por así decirlo, detalles particulares que identifican y particularizan la naturaleza de un espacio concreto.

2. La presencia y la proliferación del protestantismo en los pueblos indígenas han cumplido gran parte de su misión: desterrar las ideas en torno a los seres invisibles del paisaje que a lo largo de varios siglos han permitido a los pueblos interactuar con su medio y sobrevivir con los principios de una relación de reciprocidad - hombre-naturaleza- Sin embargo, todavía prevalece un bagaje de ideas y prácticas culturales comunitarias cuya base sigue siendo la agricultura. De este modo, incluso los protestantes más devotos guardan en su memoria la necesidad de ubicar la posición del sol y la luna antes de la siembra, la cosecha o la tala de árboles para sus casas, además de para la realización de otras actividades domésticas. Es inevitable que refieran la sacralidad de los cultivos, de la tierra y de los manantiales como elementos custodiados por un guardián que, según ellos, es enviado por el dios cristiano.

3. Por último, la negación de las personas a externar las ideas que por naturaleza son sagradas es un derecho y una responsabilidad que les permite mantener en orden y conservar la sacralidad de su discurso, de su conocimiento y de su tradición religiosa. Por otro lado, dicha negación se debe en gran medida a la desconfianza que generan las personas externas a la comunidad que llegan, dicen algunos habitantes, a saquear el conocimiento.

No obstante, pese a las dificultades enumeradas, varias entrevistas realizadas dan cuenta de valiosa información que permite conocer y entender la naturaleza de las cuevas como espacios sagrados de muy larga tradición en Chiapas. 


\section{Sobre cuevas en el área maya}

Decíamos al principio que los estudios que destacan los usos que las cuevas han tenido en Mesoamérica son prolíficos. Bonor Villarejo nos recuerda que "fue fray Diego de Landa quien nos proporcionó las primeras noticias sobre las cuevas mayas en su libro Relación de las cosas de Yucatán", y que habrían de pasar más de dos siglos para que otros interesados publicaran obras sobre cuevas, como John L. Stephens en 1843 y Henry C. Mercer en 1896. Pero es Thompson, en 1959, quien "intenta y consigue una valiosísima aproximación a la utilización de las cuevas por los mayas antiguos y contemporáneos" (Bonor, 2003: 2).

Efectivamente, Thompson sostuvo tempranamente que "Los mayas parecen haber utilizado las cuevas principalmente para ritos religiosos, como depósitos del 'agua virgen' empleada en las ceremonias, y para desprenderse de los muertos. También fueron usadas, aunque de forma limitada, como lugares de refugio y como basureros" (Thompson, 1959: 396). Bonor confirma y amplía la información acerca de estas funciones, tanto en la antigüedad como en nuestros días, y sostiene que "fueron en gran parte suministradoras de agua a los centros [de población próximos y sitios para] ceremonias de renovación anual, autosacrificios, enterramientos, obtención de zuhuy ha [agua virgen], etc." (2003: 3). Además de lo anterior, el mismo autor precisa, al asociar la cueva con el Inframundo en la cosmovisión maya, que:

las cuevas no van a ser únicamente la representación del inframundo, sino que también serán consideradas como el lugar habitado por los dioses e, incluso, la patria lejana y primera de los primeros hombres. Esta serie de circunstancias originan que las cavernas sean recintos donde los mayas prehispánicos celebraran ritos de muy variada índole. Enterramientos, incineraciones, sacrificios y manifestaciones artísticas, son el resultado de algunas de estas ceremonias (Bonor, 2003: 4-5).
Galina Yershova nos recuerda que "la misma cueva, en su concepto más arcaico, encarnaba al útero materno, dador eterno de vida. Por ello incluso la simple visita al espacio sagrado de la cueva por el hombre se identificaba con la iniciación, con una nueva etapa o con la renovación de la vida" (Yershova, 2006: 9). Finalmente, un resumen interesante de los estudios sobre cuevas en el área maya lo presenta Sheseña (2006); asimismo, un rico compendio bibliográfico sobre el mismo tema en Mesoamérica lo ofrece Brady (2009).

\section{Algunos atributos de las cuevas según la tradición oral tseltal contemporánea}

Aún es posible sostener, con datos provenientes de la observación directa y la tradición oral de nuestros días, que en la mayoría de los pueblos indígenas de Chiapas las cuevas son todavía un elemento vital de la religión doméstica o cotidiana. A través de ellas se establece la comunicación entre los hombres y las deidades, la cual tiene fines específicos, entre ellos el de solicitar abundancia de sustento y salud del alma. En el municipio de Tumbalá, la cueva de Joloniel cumple con esta función. Los habitantes acuden ahí, a la morada de don Juan, dueño de la tierra, el 3 de mayo de cada año para pedir el agua que garantiza la fertilidad de la tierra y, en consecuencia, la buena cosecha (Meneses López, 1998). En Oxchuc, la cueva artificial de la montaña Ik'al Ajaw - pues la cueva natural, según el mito local, fue rellenada por los mismos habitantes antes de que los soldados coloniales provenientes de la Capitanía General de Guatemala saquearan las riquezas de su interior ${ }^{1}{ }^{1}$ posee la misma sacralidad y las mismas funciones que cualquier otra caverna de montaña. El mito acerca de las riquezas en el interior de la montaña, además de estar extendido por todo el área mesoamericana, confirma la naturaleza simbólica de la cueva de la montaña Ik'al Ajaw: es lugar de realización de rituales y, al mismo tiempo, es vía de acceso a ese mundo de riquezas. Al menos en Oxchuc, esta creencia está generalizada y hay quienes pueden señalar nombres de personas que han obtenido los "regalos" de la montaña en forma de monedas o 
cosechas, concedidos por su guardián, el Ajaw, un anciano mestizo y prepotente.

Ahora bien, en nuestra indagación hemos identificado otros atributos de las cuevas, los cuales sólo salen a colación en conversaciones de confianza que, en este caso, sostuve en gran parte con mi padre, Pedro Méndez Oleta, en Petalcingo, Chiapas. Hemos ordenado dichos atributos, según nuestro criterio, en orden de importancia. De esta manera, la noción de kuxul indica literalmente que algo está vivo o animado; por tanto, es éste un atributo que define la naturaleza sacra de las cuevas, noción bastante extendida por otros pueblos de origen mesoamericano (Báez, 2004). No todas las cuevas de la geografía poseen la cualidad de kuxul, por amplias y profundas que sean o sirvan de refugio a cierto tipo de animales. En Petalcingo, por ejemplo, se encuentran las cuevas Sna sots' (Casa del Murciélago) y la Sna ik' (Casa del Viento), pero no son consideradas sagradas y no hay en ellas ninguna evidencia de rituales a pesar de que se cree que la primera funciona como túnel o pasadizo, de aproximadamente dos kilómetros de longitud, que conduce al otro extremo de la colina. Por el contrario, a las cercanas cuevas de Joloniel, en Tumbalá (Meneses López, 1998; Sheseña, 2008), y a la de la montaña Ajk'abalna, en Yajalón (Sánchez Carrillo, 2007), se les otorga el atributo de kuxul porque, además de situarse en el cuerpo o como parte sustancial de la montaña, se las asocia con un ser sagrado llamado Ajaw en la actual lengua tseltal. La noción de kuxul atribuida a las cuevas - así como a la tierra y las montañas - se otorga en función de este personaje, el guardián y protector de los animales y los cultivos básicos, como el maíz y el frijol. Por ello, las cuevas de esta naturaleza son consideradas también, en el lenguaje ordinario, como yajnib chanbajlam (escondite o guarida de animales) porque éstos ahí viven o se resguardan de los cazadores. En contraparte, existen cuevas que, al ser profanadas por los hombres mediante el saqueo de sus riquezas, o al haber "muerto" su guardián, ${ }^{2}$ pierden su carácter de kuxul y se vuelven cavidades simples, vacías, deshabitadas, muertas.

Para ser precisos, el Ajaw en realidad no reside en las cuevas, como se ha dicho con frecuencia. De hecho, en conversación con Pedro Méndez Oleta, éste menciona que la cueva es sólo un skuxibyo'tan Ajaw (descansadero del Ajaw) o su lugar de vigilancia y contemplación del mundo. Por ello, los jueves y viernes se convierten en los días especiales para la realización de rituales, pues es cuando el Ajaw descansa en las cuevas y llegan las plegarias directamente a sus oídos. ${ }^{3}$ Esto sugiere que reside en otro espacio más profundo de la montaña, un lugar propio para deidades y seres invisibles, llamado en tseltal yan [ye'tal] bajlumilal (otro mundo, comúnmente subterráneo) o k'atinbak (lugar de los huesos ardientes). Ese mundo subterráneo, actualmente nombrado también como "almacén" o "bodega de alimentos" o "montaña de los mantenimientos" (López y López, 2009), es una proyección al ámbito sagrado de las características de la sociedad y del paisaje, de carácter cambiante en los aspectos sociales y estático, e imperecedero en los aspectos del paisaje.

En este sentido, la cueva como "descansadero del Ajaw" es también considerada por los ancianos una stinail o sbentanail wits (puerta o ventana de la montaña), es decir, un acceso al mundo subterráneo. La cueva es la puerta de la montaña a la que el Ajaw se asoma los días jueves y viernes para vigilar y contemplar el mundo, y a la que llegan también los hombres a tocar la "puerta" con sus plegarias, su música y sus ofrendas, tal como todavía se hace en las puertas de las iglesias católicas en fechas y festividades particulares. Asimismo, la cueva es también un yich'u-ik' bajlumilal (por donde respira el mundo) o, más apropiadamente, la nariz del mundo, de la tierra, pues recordemos que ésta ha sido personificada como un anciano desde la antigüedad mesoamericana $y$, por tanto, posee la capacidad de respirar y exhalar. Aunado a esto, la cueva es también una fuente de nube y de viento, dicho en tseltal slok'ib tokal y slok'ib ik', respectivamente, como evidencias metafóricas del aliento y la respiración de la montaña. Pero sobresale principalmente su función de entrada al mundo subterráneo y de salida a la superficie terrestre para los humanos y seres invisibles, según ha sido ya suficientemente documentado por los investigadores arriba citados y muchos otros (Moyes, 2007; Manahan, 2000; Manzanilla, 1994 y otros). Esto indica que, sin la cueva, la montaña no tiene ni puede tener la cualidad de mundo subterráneo, aunque sí otro 
tipo de importancia, como el hecho de ser un marcador natural del territorio, por ejemplo.

Otro atributo de las cuevas que llama la atención, además de ser guarida de animales, es el que la describe como "cárcel" de perros cazadores que, por faltar sus dueños a la costumbre de pedir permiso al Ajaw, frecuentan el monte y hieren a los animales. Como ejemplo, don Pedro Méndez Oleta cuenta un relato cuya sinopsis es la siguiente: un hombre frecuentaba la montaña para cazar animales sin el consentimiento del Ajaw. En una ocasión, el perro quedó atrapado en la cueva y, cuando el cazador fue a buscarlo, encontró en la entrada un anciano desconocido a quien preguntó si había visto el animal; éste respondió que sí, que se encontraba encerrado dentro de la "casa" y que, si quería, se metiera a identificarlo entre tantos otros que habían sido encerrados con anterioridad. Aunque en otras narraciones se recupera el animal perdido mediante un ritual, aquí el anciano preguntó al hombre si tenía hambre y éste contestó afirmativamente. El anfitrión le dijo que dentro de la "casa" se encontraba su consorte y que le pidiera una taza de pozol. El hombre vio a un enorme sapo y se lo hizo saber al anciano; éste le aclaró que se refería a ese animal, por lo que el invitado entró de nuevo y le habló. El sapo, en su papel de esposa, lo hizo sentar sobre un armadillo, considerado asiento de los dioses. Enseguida, el anciano le mostró los animales dentro de la cueva, encerrados en un corral cuyos postes y ataduras eran serpientes. Le pidió al hombre que identificara a su perro y, después de advertirle sobre las consecuencias de otro descuido o del incumplimiento de la tradición, lo dejó salir.

En episodios de esta naturaleza ocurre con frecuencia que el personaje que ha dialogado y conocido la morada de los guardianes de la tierra muere tres días después. ${ }^{4}$ No obstante, lo que de esta narración interesa es que describe el interior de la cueva, concebida como lugar para castigar no sólo a los animales, sino también a las personas que desobedecen las normas de la tradición.

Un caso similar aconteció en la comunidad Lázaro Cárdenas, municipio de Yajalón. Don Rafael Cruz Hernández y un compañero suyo fueron un día de paseo a una de las cuevas de la montaña Ajk'abalna. Don Rafael asegura que dentro de ella vieron varios tipos de animales encerrados en un corral construido con serpientes, todos al cuidado y la conservación del Señor de la Montaña, el Ajaw. Estos animales pueden salir a la intemperie, especialmente en temporadas específicas de siembra, desarrollo y cosecha del maíz, para alimentarse. La idea de que provienen de las montañas y son custodiados por el Ajaw ha dado lugar a innumerables relatos muy elaborados. En Petalcingo se narran episodios vividos o escuchados acerca de manadas de jabalíes en las milpas, guiadas por un anciano o un mestizo, que desaparecen en la barranca de algún cerro cercano después de estropear parte del sembradío. Hay hombres que, por aparente descuido o por desconocimiento de la tradición, según se observa en el fondo de la narración, molestos porque sus maizales están siendo devastados, acuden a la cueva a reclamar los estragos en su milpa. El guardián, quien aclara que no tiene con qué compensar los daños, y justificando que sus animales necesitan alimentarse de cuanto dispone la naturaleza, ofrece uno de ellos. El hombre atrapa uno sin dificultad pero, al regresar a casa, se descubre en otro sitio bastante alejado de su lugar de origen. ${ }^{5}$

Es común también la idea de que las cuevas son casas o guaridas de los rayos blanco y verde, considerados buenos y descritos como pequeños seres que en días nublados deambulan en la superficie de las montañas y comen las puntas tiernas de las plantas de chayote; por otra parte, los rayos rojos, considerados malos y muy propensos al enojo, viven en los huecos de los árboles cerca de los manantiales. Los rayos blanco y verde habitan en las cuevas, pero no en el mundo subterráneo. En las cuevas están como guardias, según uno de los entrevistados, custodiando la "gran puerta de la montaña, que no está abierta, y que sólo es como una silueta [...] y están ahí todos los rayos, ahí están; por eso, si un rayo quiere matarte, te mata." ${ }^{6}$ Más específicamente, los rayos "se parecen a los soldados que caminan. Así son ellos, por eso cada uno tiene su arma, ellos son los guardianes" de la entrada al interior de la montaña. 
Un anciano principal de Petalcingo, Anacleto Pérez Gómez, refiere que los inquietos rayos se encuentran bajo la custodia del Ajaw; en las temporadas de lluvia son puestos en libertad y es así como puede vérseles, jugando por el cielo con sus relámpagos y sus "gritos". Al finalizar la temporada, son nuevamente encerrados en la cueva. Resulta interesante que aquí los rayos no sean en sí los dioses de la tierra, como ocurre con el Chauk (rayo) en las creencias tseltales y tsotsiles de Los Altos de Chiapas, que reside dentro de la cueva y la montaña al mismo tiempo, como anciano, rayo y espíritu de la tierra (Thompson, 2004: 326-329; Gorza, 2006: 17; Köhler, 2007), tal como se concebía en la época prehispánica (Taube, 1992; Sheseña, 2006; Bassie, 2002). En Petalcingo, y en general en los pueblos tseltales de la región Selva, el espíritu de la tierra y la personificación del rayo son, al parecer, identidades diferentes: el primero, como deidad principal y creadora, tiene bajo su control al resto de los dioses, entre ellos al rayo, visto comúnmente como un pequeño niño que requiere de su "camisa" o "capa roja" para desencadenar en retumbos la furia de sus poderes, como el rayo verde de la montaña Yaxwinik (Hombre Verde) de Chilón, considerado el hijo del anciano-espíritu que ahí reside.

De acuerdo con lo anterior, pareciera que en la región Selva de Chiapas existe la idea de que cada fuerza sobrenatural tiene su propia personificación: como el viento, que se personifica en forma de mujeres y hombres con larga cabellera, de ahí que algunas personas cuenten que en su milpa o cafetal encuentran mechones de cabello entre los ramajes; las nubes, que se personifican en forma de muchachas de piel y ropa blancas que, siendo hijas del Ajaw, parecen habitar simultáneamente en las cuevas y en el Otro Mundo; los temblores, que se deben a que los Sostenedores del Mundo, al sentir cansancio, cambian de hombro, provocando con ello un ligero movimiento; o el fuego que, en un relato narrado por Pedro Méndez Oleta, se manifiesta como una joven que se forma con las llamas de una fogata para anunciar a un viajero la llegada de sus enemigos y la manera en que podría derrotarlos.

Las cuevas también funcionan como túneles de comunicación entre un puebloy otro, entre una montaña y otra. Por ejemplo, se piensa que en el interior de la montaña Ajk'abalna, en Yajalón, existen túneles que conducen hacia otras montañas de importancia ritual: hacia Don Juan rumbo a Palenque, hacia Yaxwinik en Chilón y hacia Ik'al Ajaw en Oxchuc. ${ }^{8}$ En palabras de don Juan López, la montaña Ajk'abalna también tiene cuatro enormes raíces de oro, orientadas cada una a los cuatro puntos cardinales; por esas raíces — seguramente metáforas de túneles subterráneos- se desplazan los guardianes de la tierra cuando se comunican o se visitan entre ellos, yendo de un lugar subterráneo a otro. En la cabecera municipal de Yajalón, el señor Gustavo Gómez Méndez refiere también que ha visto en sus sueños, es decir, a través del ch'ulel, que dentro de la montaña ubicada en su terreno de cultivo, en el extremo este de la montaña Ajk'abalna, brota un manantial y se dispersa en cuatro canales subterráneos, orientados cada uno a los puntos cardinales.

En Petalcingo, donde anteriormente hemos realizado trabajos de recopilación de relatos orales, se cuentan varias versiones de una leyenda interesante acerca de una poza formada en el recodo del río Pajwuchil (Lugar del ámbar), el cual nace en las faldas de la montaña Ajk'abalna. El nombre de la poza, Ch'ayuants, significa "donde la mujer se pierde". Esta mujer es la hija mayor de la montaña en cuestión y, según la leyenda, se desplaza por debajo del río y de la tierra (Martínez, 2010: 57-80). Al poniente de la misma población, monte adentro, se encuentra una laguna llamada El Jbobo en honor al personaje mítico que la custodia. Dicho personaje también se desplaza por debajo de la tierra a través de las cuevas (Martínez, 2010: 81-93). En suma, cada sitio importante en la geografía, en particular en el interior de los ríos y manantiales o cerca de ahí, funciona como túnel que comunica con otros sitios más grandes e importantes. En este contexto, las cuevas son túneles de comunicación entre un sitio y otro, además de que conducen al mundo subterráneo dentro de la montaña.

En la actualidad, las cuevas son todavía espacios propicios para la realización de rituales agrícolas; incluso en algunas partes, especialmente en comunidades alejadas de los centros urbanos, son escenario de rituales para la restauración de las almas agredidas por naguales 
o por el guardián mismo de algún lugar profanado. En el primer caso, si bien en contados pueblos se realizan peticiones de abundancia antes de la siembra y muy pocas de agradecimiento después de la cosecha, no cabe duda de que todas ellas se han concentrado en un solo ritual de más envergadura: el ritual de la Santa Cruz, celebrado el 3 de mayo de cada año. Esta ceremonia, muy a pesar de que se encuentra incrustada en el marco de las festividades religiosas de la Iglesia católica, es eminentemente agrícola. Los elementos que la fundamentan son los relacionados no sólo con el objeto de culto y el espacio donde se realiza, sino también con el discurso mismo que los oficiantes pronuncian y la época en que se lleva a cabo. En primer lugar, la cruz funciona en estas prácticas como el árbol cósmico, la gran ceiba que comunica los tres niveles del universo por donde "las fuerzas divinas bajaban" (López, 1996: 75) y, por tanto, por donde fluyen también las plegarias humanas. El manantial donde con frecuencia se realiza es la clave para vislumbrar su naturaleza agrícola pues, al igual que la cueva, funciona como vía de comunicación hacia el interior de la montaña; en este sentido, los oficiantes del ritual dialogan con la deidad de la tierra. Está por demás decir que a partir del mes de mayo inicia el período de lluvia y es propicio para la siembra, lo que hace necesaria y sustancial la ceremonia de la Santa Cruz para el buen crecimiento y la buena cosecha, según la lógica del pensamiento agrícola. Al respecto, en su estudio sobre la cueva de Jolja' en Tumbalá, Karen Bassie sostiene que "la mayoría de celebraciones del día de la cruz incluyen aspectos de los rituales de lluvia precolombinos, debido a que el 3 de mayo coincide con el principio de la estación de lluvias, y la región ch’ol no es una excepción" (Bassie, 2006).

En cuanto al uso de las cuevas para la restauración del alma es, en la actualidad, de escasa importancia por motivos relacionados con la modernidad — venta de medicamentos, educación escolar, protestantismo, energía eléctrica-. Sin embargo, ello no indica que las prácticas rituales de curación hayan perdido credibilidad, pues los rituales de recuperación y restauración del alma del enfermo se hacen donde se supone que éste padeció un descuido o profanó un sitio, o desde la casa del paciente, haciendo uso del mismo discurso que es también un diálogo con el Ajaw, quien captura el alma del profanador. Cualquiera que sea el sitio para este ritual, el oficiante abre en la tierra un orificio en el que deposita los regalos para el Ajaw. Por tanto, este orificio funciona como una cueva, una vía de comunicación con el mundo subterráneo.

Por todo lo anterior, en su papel de entrada hacia una "casa" o un mundo subterráneo, la cueva y la montaña se convierten en un yajnib ch'uleletik (refugio o escondite de almas), almas que son perseguidas por un ch'ulel más fuerte y dañino, es decir un lab (nagual). Es en este contexto en el que el señor Anacleto Pérez Gómez, refiriéndose a la montaña Ajk'abalna, nos dice: “A veces entro ahí. Es que muchas veces me han perseguido, por eso voy ahí, entro pues y rápidamente abren la puerta, entro; llega el que me persigue, entonces sale el guardia le dan sus chicotazos al regresar." Don Anacleto entra en la montaña porque, siendo ésta un refugio, acude ahí para salvar su ch'ulel cuando un lab nocivo trata de hacerle daño. Al preguntarle acerca de quiénes pueden ser los lab enemigos, nos aclara que "son los del pueblo, los de corazones malos; es que quieren hacerle daño a los que tienen almas buenas." ${ }^{10}$

Por último, la cueva es también una barrera cósmica entre dos dimensiones distintas de tiempo y espacio, cada una con características y naturaleza propias; por tanto, la comunicación recíproca entre ambas dimensiones es a través de la cueva como punto de transformación, pues los humanos no pueden acceder al mundo subterráneo físicamente - en cuerpo y sangre- , sino sólo a través del ch'ulel-alma, y tampoco los seres del mundo subterráneo pueden manifestarse en la superficie terrestre como espíritus, sino sólo bajo la forma de animales o de seres humanos. Cada cual, al retornar a su ámbito de origen, recupera su forma y naturaleza anteriores.

Hemos ya presentado algunos ejemplos del primer caso, los cuales tratan de viajes de personas al interior de la montaña con propósitos particulares. Ahora ofrecemos un ejemplo del segundo caso, en que un Ajaw, teniendo aspecto humano dentro de la cueva y de la montaña, al salir de éstas para manifestarse en 
la superficie terrestre adquiere un aspecto animal. Al respecto, el señor Gustavo Gómez Méndez de Yajalón nos refiere que: "el Ajaw es como nosotros, pero es un anciano guardián, viejo; antes de salir de la montaña es como nosotros, un guardián, pero al salir afuera se convierte en una serpiente, se transforma en una serpiente, entonces ya sale afuera."ll

\section{Algunas formas de acceso al mundo subterráneo}

Finalmente, consideramos que no está de más enumerar algunas formas de acceder al mundo subterráneo, así como los diferentes propósitos de la gente que hace los viajes, los cuales no sólo son de carácter ritual, sino que también son ejecutados con motivo de incidentes relacionados con la vida cotidiana. En Yajalón, un relato describe cómo dos hermanos visitan una laguna y ven a una sirena en la orilla, que era una mujer lavando ropa sobre una piedra. El menor, sorprendido por su belleza y desobedeciendo las advertencias de su hermano, se acerca a la muchacha dispuesto a enamorarla:

Bajtla sk'opon. Matola sk'oponeja, japuyotbael k'alal ta yut ja'. Ma' jaymel achiknaj. Ja' te pak'etik limajtik ta k'ajk'ale malajba' chiknaj. Maba layil te wa'iy bankilale. Bajtla yil, mi ajch'emuk te ton banti la yak'sbae, mala binti ay, senyanax la yak'. Senyailnax, yu'unax ya slo'lo kristiano, ya yik' bael. Lajla smil te wa'iye, ja' la yik'bebael te xch'ulele, jil ta yutja' stukel winike. [Te bankilale] Bajtla sle, ochla bael xch'ujlele; bajtla sle ta banti ay ch'ene; ayla muk'ul ch'ena ta yut nabile, jich bin ut'il muk'ul posoyi, awil ayla chena, ochla bael xch'ulele, bajtla sle...

Se fue a hablarle. Todavía no le había hablado, le atacaron y cayó al agua. Ya no apareció nunca. Las ropas tendidas al sol desaparecieron. El hermano mayor no se dio cuenta. Lo fue a ver, no estaba mojada la piedra donde ella había estado. No había nada, sólo fue una señal. Es una señal nada más, sólo para engañar personas y se las lleva. A aquél dicen que lo mató, le llevó su alma. El cuerpo del hombre, en cambio, quedó dentro del agua. [El hermano mayor] fue a buscarlo, su alma se metió a buscarlo en una cueva. Dicen que hay una gran cueva dentro de la poza, es como una gran laguna; entonces hay una cueva en su interior, su alma se metió por allí, fue a buscarlo. ${ }^{12}$

De acuerdo con la cita anterior, los portales de acceso al mundo subterráneo existen tanto en la superficie como en la profundidad de ríos, lagunas y manantiales. Un cuento zoque, cultura que comparte elementos comunes de su cosmovisión con los diferentes pueblos mayas contemporáneos, también da cuenta de cómo las cuevas cumplen con la función ya referida. El relato trata de un campesino que viaja a las faldas del volcán Chichonal en busca de barbasco; fracasa en su primer intento y decide volver al mismo sitio el segundo día, pero:

su sorpresa fue tan grande, ya que donde había buscado encontró el barbasco, en la superficie, como si fuese tronco tirado; lo único que hizo fue cortar y llenar su red, ponerle mecapal y regresar temprano. Pero por curiosidad fue a cerciorarse dónde quedaba ese sitio y recorrió un buen tramo, encontrándose una cueva entre un peñasco; se aventuró en el interior: se sentía atraído por el sitio, era silencioso y tenía una buena temperatura. Entonces se preguntó: "iPor qué estará caliente?” De pronto vio un claro en el fondo de la cueva y decidió ver a dónde llegaba; se hizo más notable la claridad y de pronto una voz lo sobresaltó: "Bienvenido, pasa"; era una voz de hombre. Tras abrir una puerta metálica, repentinamente se encontró con un pueblo (Gómez Martínez, 2002: 138).

El personaje recorre el pueblo situado dentro del volcánmontaña; al regresar al suyo, en la superficie, encuentra que la gente lo andaba buscando con cohetes y tambor y, al tercer día, muere sin causa aparente.

Un relato tsotsil da cuenta de un caso similar. Se trata de un hombre huérfano que padecía hambre con su mujer y su hijo. En el monte, a la orilla de un camino, se sentó a llorar cuando pasó un ladino montado en su caballo que le ofreció trabajo. Después de que se pusieron de acuerdo: 
el hombre se fue con el ladino, se fue pegado al caballo. Le dijeron que cerrara sus ojos, y los cerró, y cuando los abrió ya estaba en la casa del ladino, aquél era el dueño de la tierra que estaba cerca de donde vivía aquel hombre, la mujer estaba esperando y esperando que llegara su esposo y no llegó.

Ahí se encontraba toda triste, porque no tenía con qué alimentarse y ella se fue a buscar su leña, como lo hacía siempre su esposo. Cambiaba leña por tortillas, y así siguió viviendo la mujer con su criatura.

Entonces el hombre tuvo la oportunidad de trabajar con el señor que era el dueño de la tierra; el oficio que le dieron fue el de arriero.

Se llevó los caballos, que cargaban puras monedas, eso era para la paga de los trabajadores en otro pueblo. De ahí le dieron un par de huaraches de puro fierro y le dijeron:

- Vas a regresar cuando se acaben tus huaraches (De la Torre, 1997: 148).

En esta narración, el hecho de cerrar los ojos al viajar en el lomo de un caballo se realiza para cruzar hacia el mundo subterráneo. Aunque no se habla precisamente de una cueva como portal de acceso, la idea está implícita puesto que no existe, al parecer, otra vía para tal propósito. Aquí, la presencia del ladino es una reminiscencia de pasajes y personajes históricos, como la época de los ladinos finqueros que reconfiguraron las visiones locales del mundo. Estos ladinos, que en otras versiones son dos, uno el que transporta y otro el dueño del lugar, fusionaron sus aspectos con los del espíritu-guardián de la tierra mediante procesos de resemantización o revalorización. Por ello, los atributos y las funciones de este espíritu-guardián, además de los de carácter agrícola, coinciden —en rasgos, actitudes y pertenencias- con los de los finqueros que abundaron en Chiapas durante el siglo XIX y la primera mitad del XX. En este sentido, el ser sobrenatural que ayuda a cruzar la frontera cósmica representa a los enganchadores que iban de paraje en paraje para reclutar hombres como mano de obra barata en las fincas. El calzado de metal que el guardián del lugar da al recién llegado representa las deudas que asumían los enganchados, quienes estaban obligados a saldarlas antes de regresar a casa. ${ }^{13}$ En suma, el relato muestra una faceta resemantizada de la cosmovisión tsotsil contemporánea, pues la creencia, como producto social, está impregnada de pasajes históricos que sus portadores han sabido amalgamar creativamente inspirados por su medio geográfico, específicamente por las cuevas y las montañas. Aunado a esto, ya sea por la proyección del carácter tormentoso de las fincas o por influencias de la Iglesia católica, el mundo subterráneo es un espacio donde hombres y mujeres, después de la muerte, transforman su condición humana en otra: se convierten en animales de carga. Por ejemplo, en el relato de Méndez Guzmán (1996), un hombre de Tenejapa viaja al fondo de la tierra en busca de su mujer fallecida y la encuentra convertida en una yegua de carga al servicio del guardián-finquero de ese lugar. Esto indica que la asimilación de nuevas categorías culturales, en particular las derivadas del cristianismo católico y reflejadas en la religiosidad tan arraigada y profunda, incluso en las actividades y actitudes más comunes de la gente, hace que la experiencia cotidiana se desplace entre lo humano y lo divino; por tanto, el interior de la montaña viene a funcionar como un purgatorio, un espacio de tormento y transición hacia el descanso eterno junto a los ancestros.

El viaje a través de las cuevas se logra con la ayuda de un ladino y un perro negro que funge como un caballo (Meneses Méndez, 1998); o bien se hace a pie, como el hombre de Tenejapa que, al morir su esposa, decide buscarla en el K'atinbak (lugar de los huesos ardientes). Para llegar a ese lugar:

Caminó mucho, sin pensar siquiera en el tiempo, pues la oscuridad y el silencio temibles le habían nublado la mente de tal manera que andaba sin darse cuenta, sólo unido al recuerdo de su esposa. De pronto notó que el camino se terminaba y se abrió ante sus ojos un anchísimo pozo tan profundo que parecía no tener fin. Aterrado, pensó que no habría manera de bajar por sus paredes, pero tanteando halló que un camino ancho y fácil bajaba en espiral hasta el fondo. No supo cuánto tiempo descendió, hasta que alcanzó 
a ver una débil luz, lo que le dio nuevas fuerzas, pues pensó que podría provenir de algún lugar habitado (Méndez Guzmán, 1996: 64). ${ }^{14}$

En Oxchuc se tiene una idea similar. La entrada al interior de la montaña Ik'al Ajaw es la cueva artificial que parece sólo una hondonada como de cinco metros de profundidad, cavada en tiempos coloniales porque, según el mito local, la cavidad natural fue cerrada con piedras para evitar que los soldados provenientes de Guatemala saquearan las monedas de oro que había en su interior. ${ }^{15}$ Sin embargo, las barrancas de sus costados y alrededores funcionan también como portales de acceso. De hecho, de acuerdo con el señor Mariano Sántiz, principal de la iglesia de Santo Tomás, el guardián de la montaña se manifiesta en persona cuando el silencio asola la intemperie para rondar a los que, desobedientes y faltos de conocimiento de la tradición, hacen perjuicios contra la naturaleza. ${ }^{16}$

Otra manera de entrar en el mundo subterráneo es a través de los sueños. Es decir, el ch'ulel sale del cuerpo por las noches a recorrer el espacio que corresponde a su naturaleza. Se trata de las "almas" — compañeroanimal o meteoro-, fuertes e inteligentes como los rayos verde y blanco, que pueden penetrar sin dificultad en las entrañas de las montañas a través de sus puertas o ventanas: las cuevas. En caso contrario, si el ch'ulel de la persona es débil o poco inteligente, se enfrenta a dificultades que le impiden recorrer el lugar. Esto es lo que podemos inferir de la experiencia onírica del señor Francisco Méndez Pérez, de Petalcingo, quien asegura que la montaña Ajk'abalna, situada a escasos kilómetros del poblado:

Mero na. Komo ay kiloj ta kwayich euk. Pero ja'nax yu'un maba, maba jamboton komo ma'yuk suerte ku'un ya'yel ya $x$-ochon tey ejka (Eso). La kna' awijlon bael, k'ajon li'nix a jajchon ta klumaltiki; la kna' xjayeton bael te'a. K'oon teya, jich but'ik yoyal ajensiai, jich ja'chuk ay yoyal ta skoridor sjoylajal [...] Jich. Och kjojyobta me ta kwayiche. Personal k'oon ta beela. Xtejk'lejon k'oel ta sti'il. Pero niwak pilastre sjoylejal, och kjojyobta. Pe spisil makal me ti'nae. Ma'yuk ba' je'el la kta... wen material na me ta kwayiche. Ma'a, ma' witsuk, ma'a joch binax jich wits ya xk'ootik tey ae. Mero ja'nix jich but'ik sna rikoetiki.

Es mera casa. Porque la he visto yo también en mi sueño. Pero sólo que no me abrieron [la puerta] porque no tengo suerte pues para entrar. Lo supe cuando fui volando, parece que aquí comencé [el vuelo] en nuestro pueblo; lo supe cómo fui fugazmente allá. Llegué ahí, así como los pilares de la agencia, asimismo tiene pilar alrededor del corredor [...] Sí. Comencé a recorrer a su alrededor, en mi sueño. Llegué a caminar ahí personalmente. Llegué pronto a pararme a la puerta. Pero a su alrededor habían grandes columnas, comencé a caminar a su alrededor. Pero todas las puertas estaban cerradas. Por ningún lado la encontré abierta. La casa era de puro material, en mi sueño. No, no es un cerro, no podemos llegar ahí así nada más como a cualquier cerro. Así es como la casa de los ricos. ${ }^{17}$

De acuerdo con la cita anterior, el no haber tenido acceso al interior de la "casa", como el mismo entrevistado llama a la montaña en cuestión, se debe en parte a la debilidad atribuida a su "alma". En este sentido, las personas comunes no están facultadas para cruzar la barrera cósmica salvo si tienen un objetivo muy concreto que les permita enseñar a los demás parte de esa "casa”. No pueden, en teoría, entrar ahí a su voluntad y, si lo logran, es por casualidad, por lo que difícilmente distinguen caracterizaciones sustanciales - porque llegan ahí por descuido o sin suerte- y no saben, al parecer, conducirse por los caminos de ese mundo subterráneo.

\section{Palabras finales}

Los estudios han resaltado que las cuevas son espacios sagrados por excelencia, idóneos para todo tipo de rituales públicos y privados, políticos, agrícolas y de sanación, pues forman parte de un espacio más amplio y estructurado, la montaña, considerada como el lugar, la fuente o el almacén de los alimentos, y la residencia de los dioses y de los espíritus de los hombres y la naturaleza. En este sentido, la importancia ritual y simbólica de las 
cuevas está relacionada con la montaña de la que forman parte. A esto agregamos que, siendo la montaña una réplica del universo, ésta contiene en la parte profunda de su base un espacio idealizado, el mundo subterráneo, cuyas caracterizaciones geográficas, y sobre todo la dinámica de operación e interacción de sus habitantes, imitan las existentes en la superficie de la tierra.

La vía de comunicación e interacción entre ambos mundos es la cueva en su papel de puerta o ventana de las montañas y, con menos frecuencia, también lo son los sitios de importancia ritual dispersos en el paisaje, como los manantiales. La cueva es entonces para la montaña lo que la puerta para la casa, porque la montaña posee un interior que ha sido imaginado precisamente como una "casa" y como un espacio más amplio y organizado: el inframundo. Por tanto, las cuevas son conductos imaginarios hacia el vientre de la montaña, donde también habitan o se refugian animales míticos, como la popchan (serpiente petate) y la xulubchan (serpiente con cuernos) ${ }_{1}^{17}$ y reales, como los murciélagos, golondrinas, armadillos o tepezcuintles, así como seres invisibles y nocturnos, como los llamados en tseltal jow y xpajk'inte'. ${ }^{19}$

De acuerdo con lo anterior, las cuevas siguen cumpliendo una importante función en la vida cotidiana de amplios sectores de Chiapas y en general de los que son descendientes de las antiguas culturas mesoamericanas. A pesar de que estas concepciones compiten con nuevas creencias religiosas - protestantes-, para un amplio sector de la población, el agrícola o campesino, las cuevas son todavía consideradas el camino hacia el interior de las montañas, de donde proviene el sustento humano. Esta cualidad las convierte en el espacio idóneo para los rituales agrícolas y de curación. Además, con el apoyo de los conceptos teóricos aplicados, el de la racionalidad y el de la revalorización de las categorías culturales, hemos evidenciado nuevas nociones presentes en la tradición oral actual y en la intimidad de las conversaciones ordinarias. Dichas nociones, la cueva como puerta o ventana del mundo, como descansadero del Ajaw, como nariz del mundo o de la tierra, o como refugio de almas y animales, contribuyen a comprender mejor la naturaleza de las cuevas en las concepciones actuales de los pueblos tseltales de Chiapas, concepciones que resultan de revalorizaciones constantes y, por tanto, cambian de sentido y significado en particulares situaciones sociales y períodos históricos. De este modo, las nociones relacionadas con las cuevas, aunque todavía éstas mantienen ciertos matices de una "racionalidad prehispánica", también contienen rasgos de los fenómenos sociales globales más recientes que, por un lado, enriquecen y actualizan la antigua naturaleza de las cuevas en sectores específicos como el agrícola y, por el otro, deterioran y condenan al olvido sus nociones y funciones, como ocurre entre las nuevas generaciones de tseltales chiapanecos.

\section{Notas}

${ }^{1}$ A la mayor parte de las montañas de carácter sagrado se les ha considerado desde la antigüedad hasta nuestros días como fuente o bodega de riquezas materiales y simbólicas. Entre las riquezas materiales destacan ídolos de piedra o jade, estalactitas, ámbar, monedas de oro y plata o armas de fuego; y entre las simbólicas, los espíritus de plantas y animales, la salud del cuerpo y el alma, o la enfermedad. Todo ello bajo el dominio de un guardián, el Ajaw. La adquisición de cualquiera de estas riquezas se logra mediante la realización de rituales. El saqueo constituye una profanación de la cueva y la montaña y puede costarle la vida al profanador, o simplemente el Ajaw migra a otro sitio donde sea "respetado". Por otro lado, la creatividad colectiva de los pueblos se refleja en su capacidad para asociar los hechos históricos con la voluntad de las divinidades: la presencia de los soldados guatemaltecos en la montaña Ik’al Ajaw es el registro de la persecución del ejército indígena sublevado en 1712, que se dispersó por la región que abarca Cancuc, Chenalhó, Huixtán y Oxchuc, precisamente en Los Altos de Chiapas. La presencia del ejército no necesariamente tenía por objetivo saquear riquezas de la montaña, pero sí, en cambio, perseguir a los sublevados por cuevas y barrancas de la región. Sin embargo, el solo hecho de penetrar las entrañas de esos lugares, y más por un ejército acostumbrado al pillaje, no podía prevenirse de otro modo más que evitando la entrada para salvar así el "preciado tesoro". 
${ }^{2}$ En la región Selva de Chiapas se cuentan leyendas en las que los guardianes de espacios sagrados son ofendidos o maltratados y deciden cambiar de sitio llevándose a cuestas los espíritus de plantas y animales bajo su custodia. En otras, algún guardián captura el ch'ulelalma de una persona y los familiares de ésta, haciendo uso de los poderes de sus naguales de rayo, atacan y dan muerte al Ajaw.

3 Sin duda hay aquí alguna influencia de las ideas católicas de Semana Santa, sobre todo acerca de la muerte y resurrección de Jesucristo, precisamente los días jueves, viernes y sábado. No sólo porque, con la muerte de Cristo, se abre y tiembla la tierra y resucitan muertos, sino que Él mismo sale resucitado de una cueva, literalmente hablando, además de que durante su estancia en la tumba recorrió, dicen, el infierno y salvó almas inocentes.

${ }^{4}$ Relatos similares se encuentran en Meneses López (1998: 237-238), Gómez Gutiérrez (1997: 127-130), Xilón (1997: 167-173) y Méndez Maldonado (1997: 21121), entre otros autores.

${ }^{5}$ Entrevista a Pedro Méndez Oleta, Petalcingo, Chiapas, octubre de 2008.

6 "Smuk'ul puerta te witse; pe ja'nax ma' jamal, y ja'nax seña ay yilel..., y te'ay ejka spisil ja'me chajwuketik a kaltike (Ahtea'yika), te'ayika; ja' yu'un teme chajwuk ya sk'an a smilat, ya smilat." Entrevista a Marcos Sánchez Demesa, Chilón, 2008.

7 "Jich but'il jun soldadoetik yilel a xbeeniki (Ah jich). Jich ayik ejk ja'meto, ja' yu'un yajtalal stujk'ik ek; ja' jkananetik ek ja'meto." Entrevista a Marcos Sánchez Demesa, Chilón, 2008.

${ }^{8}$ Entrevista al principal Juan López Cruz, Petalcingo.

9 “Ay ya x-ochonbaela. Yax-ochonbaela. Yu'un bayel klo'oj nutsel me tey ya xboon ae, awil x-ochon bael, ora xjamot puertae, $x$-ochon bael, awil xk'ot mejnutsawe, awil xlok'ixtala mewardiae, xjapuyotixtal ta chikotelaja. Jujum." Entrevista a Anacleto Pérez Gómez, Petalcingo, Chiapas, 2006.

10 "Ja'nix te klumaltik ya'yel te mach'a bojl yo'tane, yu'un ja' yo'tan a kyuts'in te mach'a lek xch'ujlele."

11 "Ja' te ajaw, jich bit'il jun, jich bit'il jo'otik; pero kanan mamal, mamal; pero jich te bi' ora xlok'ik ta, ta yutil te witse, jich bit'il jo'otik, jkanan; pero bi' ya xlok'e, ya xlok' talele sujt ta chan; ya sujt ta chan, jich bit'il chan ya sujt, entonce ya xlok'ix talele". Entrevista realizada enYajalón, 2008.
${ }^{12}$ Entrevista a Francisco Méndez Pérez, 2007.

${ }^{13}$ Para un panorama general del contexto de las fincas en Chiapas, léanse, entre otros, a Toledo (1996, 2002), García de León (1979), Reyes (1992), Pinto (1999) y De Vos (2010).

${ }^{14}$ Una descripción similar ofrece un relato de Leticia Méndez, también de Tenejapa: "Entraron dentro de la cueva y vieron que en el interior había una puerta muy grande, que estaba abierta, aparte de las otras puertas que estaban en la entrada; intentaron entrar por ella, pero se cerró de inmediato y ya no pudieron pasar; tan sólo pudieron alcanzar a ver que había muchas personas más adentro que se estaban bañando, lavando; muchas mujeres estaban tejiendo, otras estaban peinándose, parecía como si todos estuvieran muy contentos" (Méndez Intzin, 2001: 110). En San Juan Chamula se cuenta un relato semejante al del hombre de Tenejapa (Pérez, 1997: 58-61).

${ }^{15}$ Ver nota 1.

${ }^{16}$ Entrevista a Mariano Sántiz, 18 de mayo de 2008.

${ }^{17}$ Entrevista a Francisco Méndez Pérez, Petalcingo, 2008.

${ }^{18}$ Los animales míticos como la popchan y la xulubchan protagonizan mitos locales acerca del reordenamiento constante del mundo. El primero se cree que provoca inundaciones de ríos para devastar pueblos, pero el segundo, junto con otros como la akuxachan (serpiente aguja) y la tejerexchan (serpiente tijera), abren el cauce y evitan así la muerte de la humanidad. Al menos, anécdotas de esta naturaleza salen a colación cuando, en temporadas de lluvias intensas, el río se desborda hasta ocasionar serios estragos (Méndez Aguilar, 2007). ${ }^{19}$ El Jow es un pequeño ser invisible que, según la mitología local, tiene los pies al revés y anda solitario en el monte por las noches. Gusta imitar el grito y el ajetreo de la gente, en particular de los cazadores y leñadores. La creencia dice que, si alguien pisa la huella de sus pasos, pierde el sentido y deambula como hipnotizado por el monte durante varios días hasta que lo encuentran en alguna parte lejos del pueblo. El término jow proviene del verbo jowijel (estar sonámbulo, extraviarse, perderse). La Xpajk'inte' dicen que se trata de una mujer irresistiblemente bella que viste a la usanza regional; se aparece a los trasnochados 
en lugares oscuros y apartados. Según la creencia, los hombres que la siguen amanecen en el monte, con la ropa y la piel rasguñadas y ensangrentadas. Al igual que el Jow, que se suele confundir con el Sombrerón, también a la Xpajk'inte' se la confunde con la famosa Llorona. No tenemos una traducción al español del término Xpajk'inte'.

\section{Referencias bibliográficas}

Austin, Patricia (2004), Proyecto de cuevas rituales de Belice, s.l. FundaciónparaelAvancedelosEstudiosMesoamericanos. 〈http://www.famsi.org/reports/951l0es/951l0esAustin01. pdf $>$ [10 de mayo de 2013].

Báez, Lourdes (2004), "El espacio sagrado de los nahuas de la sierra norte de Puebla", en Perspectivas Latinoamericanas, núm. 1, Japón: Nazan University. 〈http://www.ic.nanzan-u.ac.jp/LATIN/kanko/ PL/2004PDF/3lourdesbaez.pdf> [10 de mayo de 2013].

Bassie-Sweet, Karen (2002), The Creators God, s.l: Mesoweb.<http://www.mesoweb.com/features/ bassie/CreatorGods/CreatorGods.pdf> [10 de mayo de 2013].

Bassie-Sweet, Karen (2006), El proyecto de la cueva de Jolja', s.l.: Fundación para el Avance de los Estudios Mesoamericanos. 〈http://www.famsi.org/ reports/00017es/00017esBassie01.pdf> [10 de mayo de 2013].

Bonor Villarejo, Juan Luis (2003), Caves Branch Caves, Distrito de El Cayo, Belice. Reporte arqueológico de campo, s.l.: Fundación para el Avance de los Estudios Mesoamericanos. 〈http://www.famsi.org/ reports/96044es/96044esBonor0l.pdf> [10 de mayo de 2013].

Bonor Villarejo, Juan Luis e Ismael Sánchez y Pinto (1991), "Las cavernas del municipio de Oxkutzcab, Yucatán, México: nuevas aportaciones", en Mayab, núm. 7, pp. 36-52. «dialnet.unirioja.es/descarga/articulo/2774936. pdf $>$ [10 de mayo de 103].

Bonor Villarejo, Juan Luis y Carolina Martínez Klem (1992), "Traducción y comentarios al artículo de J. Eric Thompson 'The Role of Caves in Maya Culture", en Boletín Americanista, núm. 42-43. 〈http://www. raco.cat/index.php/boletinamericanista/article/ viewFile/98601/146198> [10 de mayo de 2013].

Brady, James E. (2009), Studies in Mesoamerican Cave Use: Sources for the Study of Mesoamerican Ritual Cave Use, Los Ángeles: University of South Florida/Department of Anthropology-California State University.

Brady, James E. y Juan Luis Bonor Villarejo (1993), "Las cavernas en la geografía sagrada de los mayas", en M.J. Iglesias y F. Ligorred (eds.), Perspectivas antropológicas en el mundo maya, Madrid: Sociedad Española de Estudios Mayas. «dialnet.unirioja.es/descarga/ articulo/2775805.pdf $>$ [10 de mayo de 2013].

De la Torre López, Juan (1997), "Un huérfano que se hizo rico”, en Cuentos y relatos indígenas, vol. 6, San Cristóbal de Las Casas, Chiapas: CIHMECH-UNAM.

De Vos, Jan (2010), De lejos vienen los torrentes. Una historia de Chiapas, Tuxtla Gutiérrez, Chiapas: CONECULTA.

García de León, Antonio (1997), Resistencia y utopía. Memorial de agravios y crónica de revueltas y profecías acaecidas en la Provincia de Chiapas durante los últimos quinientos años de su historia, Tuxtla Gutiérrez, Chiapas: Ediciones Era.

Gómez Gutiérrez, Enrique (1997), "El cazador", en Cuentos y relatos indígenas, vol. 6, San Cristóbal de Las Casas, Chiapas: CIHMECH-UNAM.

Gómez Martínez, Abraham (2002), “iTe’ tzitzun ngtzüjk ju'schebure jojmo?/¿Cómo es por dentro del volcán Chichonal?", en Historia y vida de nuestros pueblos. Tradición oral y narrativa indígenas, vol. 1, San Cristóbal de Las Casas, Chiapas: PROIMMSE-IIA-UNAM.

Gorza, Piero (2006), Habitar el tiempo en San Andrés Larráinzar, Paisajes indígenas de los Altos de Chiapas, México: UNAM-COLMICH.

Köhler, Ulrich, (2007), "Los dioses de los cerros entre los tzotziles en su contexto interétnico", en Estudios de Cultura Maya, vol. XXX, México: UNAM, pp. 139-152. López Austin, Alfredo (1996), Los mitos del tlacuache, Caminos de la mitología mesoamericana, México: UNAMInstituto de Investigaciones Antropológicas.

López Austin, Alfredo y Leonardo López Luján (2009), Monte sagrado-Templo mayor, México: INAH/UNAM.

Manahan, Kam (2000), Después de la caída: estudiando la naturaleza del colapso del Clásico Maya en Copán, Honduras. 
s.l.: Fundación para el Avance de los Estudios Mesoamericanos. 〈http://www.famsi.org/reports/98 038es/98038esManahan0l.pdf> [10 de mayo de 2013]. Manzanilla Naim, Linda (1994), "Las cuevas en el mundo mesoamericano", en Ciencias, núm. 36, octubrediciembre. 〈http://www.revistas.unam.mx/index.php/ cns/article/view/11419> [10 de mayo de 2013].

Martínez López, Gonzalo (2010), "Ch'ayuants” y "Mamal Jbobo”, en Marceal Méndez Pérez, Sk'oplal jo’eb cholbil k'opyu'un sk'op sti' jtseltal me'iltatiletik/Glosas a cinco relatos de la tradición oral tseltal, Tuxtla Gutiérrez, Chiapas: CELALI-CONECULTA.

Méndez Aguilar, Florentino (2007), "Ajaw ants sok te xch'ulel jtul kerem/La mujer ajaw y el alma de un joven" y "Te slab Jk'ankujketik ya smakik muk'ul ja'/ Naguales de K'ankujk bloquean el río", en Marceal Méndez Pérez, K'opti'il Yu'un woje sok yo'tik/Memorias de ayer y hoy, Tuxtla Gutiérrez, Chiapas: CELALI/CDI/ CONECULTA.

Méndez Guzmán, Diego (1996), "El K'atin bak”, en Carlos Montemayor (coord.), A'yejetik yu'un jtzeltaletik ta Tenejapa, Relatos tzeltales de Tenejapa, México: INI.

Méndez Intzin, Leticia (2001), "La niña y la cueva de los espíritus", en Sk'oplal sok snopojibal a'yejetik yu’un te tseltaletik ta stoylejal slum sk'inal Chyapa/Cuentos y teflexiones tseltales de los Altos de Chiapas, San Cristóbal de Las Casas, Chiapas: Sna Jtz'ibajom Cultura de los Indios Mayas.

Méndez Maldonado, Julián (1997), "Historia de don José y el Señor de la cueva", en Cuentos y relatos indígenas, vol. 6, México: CIHMECH-UNAM.

Meneses Méndez, Domingo (1998), "Xu'ok", en Cuentos y relatos indígenas, vol. 7, San Cristóbal de Las Casas, Chiapas: CIHMECH-UNAM.

Meneses López, Miguel (1998), K'uk'Wits, Cerro de los Quetzales. Una aproximación a la cultura ch'ol, Tuxtla Gutiérrez, Chiapas: Gobierno del Estado de Chiapas/ CELALI/CONECULTA.

Moyes, Holley (2007), Cambios y continuidades de la práctica ritual en la cueva de Chechem Ha, Belice. Informe sobre las excavaciones realizadas durante la temporada de campo 2003, s.l.: Fundación para el Avance de los Estudios Mesoamericanos. 〈www.famsi.org/
reports/02086es/02086esMoyes0l.pdf> [10 de mayo de 2013].

Page Pliego, Jaime Tomás (1996), Religión y política en el consumo de prácticas médicas en una comunidad tzotzil, San Cristóbal de Las Casas, Chiapas: IEI-UNACH.

Pérez López, Enrique (1997), Chamula, un pueblo tzotzil, Tuxtla Gutiérrez: Gobierno del Estado de Chiapas/ CONECULTA/CELALI.

Pinto Durán, Astrid Maribel (1999), Los artificios de la fidelidad. Reciprocidad y poder en una finca de Los Altos de Chiapas, Anuario 1999 del Centro de Estudios Superiores de México y Centroamérica, Tuxtla Gutiérrez, Chiapas: UNICACH.

Reyes Ramos, María Eugenia (1992), EL reparto de tierras y la politica agraria en Chiapas, 1914-1988, México: UNAM.

Sánchez Carrillo, Óscar (2007), "Cuerpo, ch’ulel y lab: Elementos de la configuración de la persona tseltal en Yajalón, Chiapas", en Revista Pueblos y Fronteras Digital, núm. 4, San Cristóbal de Las Casas, Chiapas: PROIMMSE-UNAM. 〈http://www.pueblosyfronteras. unam.mx/a07n4/pdfs/n4_art07.pdf> [10 de mayo de 2013].

Sahlins, Marshall (1997), Islas de historia. La muerte del capitán Cook. Metáfora, antropología e historia, Barcelona: Gedisa.

Sheseña, Alejandro (2006), Pinturas mayas en cuevas, Tuxtla Gutiérrez, Chiapas: CONECULTA.

Sheseña, Alejandro (2008), "Don Juan: una divinidad de raíces prehispánicas entre los actuales ch'oles de Chiapas", en Alejandro Sheseña, Sophía Pincemin Deliberos y Carlos Uriel del Carpio Penagos (coords.), Estudios del patrimonio cultural de Chiapas, Tuxtla Gutiérrez, Chiapas: UNICACH.

Taube, Karl A. (1992), The Major Gods of Ancient Yucatan, Washington: Dumbarton Oaks Pre-Columbian Art and Archaeology Studies Series.

Thompson, J. Eric (1959), "The Role of Caves in Maya Culture", en Mitteilungen aus dem Museum für Volkkerkunde in Hamburg, vol. XXV, Hamburgo, pp.122-129.

Thompson, J. Eric (2004), Historia y religión de los mayas, México: Siglo XXI. 
Toledo Tello, Sonia (1996), Historia del movimiento indígena en Simojovel, 1970-1989, San Cristóbal de Las Casas, Chiapas: IEI-UNACH.

Toledo Tello, Sonia (2002), Fincas, poder y cultura en Simojovel, Chiapas, San Cristóbal de Las Casas, Chiapas: PROIMMSE-IIA-UNAM/IEI-UNACH.

Winch, Peter (1979), "Para comprender a una sociedad primitiva”, en Alteridades, año l, núm. 1, México: UAM-
Iztapalapa. 〈http://investigacion.politicas.unam.mx〉 [10 de mayo de 2013].

Xilón Gómez, Francisco (1997), "Los tres cazadores", en Cuentos y relatos indígenas, vol. 6, San Cristóbal de Las Casas, Chiapas: CIHMECH-UNAM.

Yershova, Galina (2006), "Prólogo", en Alejandro Sheseña, Pinturas mayas en cuevas, Tuxtla Gutiérrez, Chiapas: CONECULTA. 